\title{
Stochastic Schrödinger equations with coloured noise
}

\author{
A. Barchiell:* \\ Politecnico di Milano, Dipartimento di Matematica \\ and Istituto Nazionale di Fisica Nucleare, sezione di Milano \\ C. Pellegrin讨 \\ Laboratoire de Statistique et Probabilités, Université Paul Sabatier, \\ 118, Route de Narbonne, 31062 Toulouse Cedex 4, France \\ F. Petruccione丹 \\ Quantum Research Group, \\ School of Physics and National Institute for Theoretical Physics, \\ University of KwaZulu-Natal, Private Bag X54001, \\ Durban 4000, South Africa
}

\begin{abstract}
A natural non-Markovian extension of the theory of white noise quantum trajectories is presented. In order to introduce memory effects in the formalism an Ornstein-Uhlenbeck coloured noise is considered as the output driving process. Under certain conditions a random Hamiltonian evolution is recovered. Moreover, non-Markovian stochastic Schrödinger equations which unravel non-Markovian master equations are derived.

PACS numbers: 42.50.Lc, 03.65.Ta, 03.65.Yz
\end{abstract}

Recently, stochastic wave function methods for the description of open quantum systems have received considerable attention [1, 2, 3]. These approaches have been mainly motivated by the continuous measurement description of detection schemes in quantum optics, namely direct photo detection, homodyne and heterodyne detection. In general, selective, indirect and continuous quantum measurements allow for a description of the open quantum system in terms of the stochastic evolution of its own wave function. Typically, the random trajectories of the state vector involve quantum jumps or diffusion processes and are described by stochastic differential equations for the wave function $\left|\psi_{t}\right\rangle$.

The relationship to the more traditional approach to open quantum systems in terms of Master equations for the density matrix $\rho_{t}$ is easily established by realizing that the latter can be expressed as $\rho_{t}=\mathbb{E}\left[\left|\psi_{t}\right\rangle\left\langle\psi_{t}\right|\right]$, where $\mathbb{E}$ denotes the ensemble average over realizations of the stochastic process $\left|\psi_{t}\right\rangle$. Thus, the Master equation evolution can be reproduced by generating a large number of trajectories of the state vector. This unravelling procedure, called Monte Carlo wave function method, has gained considerable importance for the numerical simulation of complex open systems [3].

The procedure described above has been a major breakthrough in the description of the Markovian dynamics of open quantum systems, where it has found many applications. At present an active line of research is to find generalizations of the stochastic Schrödinger equa-

\footnotetext{
*Electronic address: Alberto.Barchiellli@polimi.it

${ }^{\dagger}$ Electronic address: clement.pellegrini@math.univ-toulouse.fr

${ }^{\ddagger}$ Electronic address: petruccione@ukzn.ac.za
}

tion in the non-Markovian regime, which have a sound physical interpretation.

Essentially, two strategies have been investigated to accomplish this goal. A first strategy is to start from non-Markovian master equations and to try to construct pure state unravellings. It is well-know that nonMarkovian master equations can be constructed with the help of projection operator techniques. For example, time-convolutionless (TCL) projection techniques yield master equations that are local in time and allow for suitable unravellings (3, 4] for details). In the same spirit, non-Markovian generalizations of the Lindblad theory [5] have been proposed and shown to have corresponding non-Markovian unravellings [ 6, , 7]. The measurement interpretation of these unravellings is still an open problem. However, special cases of unravellings with a clear measurement interpretation have been shown to exist for discrete time models of repeated interaction [8].

The second strategy is first to generalize directly the stochastic Schrödinger equation and secondly to show if it provides an unravelling of a suitable Master equation and if it has a measurement interpretation. In [4, 8, 9, 10, 11, 12] starting from a measurement scheme a rigourous non-Markovian random evolution has been derived. The question of the possibility to express this random evolution in terms of pure states in the spirit of unravelling is still highly debated [9, 10]. For the usual scheme of indirect quantum measurement it has been shown that in general such an interpretation is not accessible [8, 10]. Other investigations promise to get unravelling [8, 9, 13].

In classical statistical physics the most straightforward way to introduce memory effects is to consider coloured noise [14]. This approach has already been successfully transferred to the theory of isolated quantum systems, 
by introducing coloured noise in the Hamiltonian of the evolution 14, 15, 16, 17]. In this paper we show how to introduce memory effects in the stochastic Schrödinger equation with the help of the addition of coloured noise. Specifically, we will illustrate the approach by using the Ornstein-Uhlenbeck process.

The alternative strategy, proposed here, is focused on an extension of the canonical Markovian approach. To be precise, generic Markovian stochastic Schrödinger equations are justified by the use of a linear equation for a non normalized state $\psi_{t}$ of the following form

$$
\mathrm{d} \psi_{t}=A \psi_{t} \mathrm{~d} t+B \psi_{t} \mathrm{~d} X_{t}
$$

where the formal derivative of $\left(\dot{X}_{t}\right)$ is a white noise, that is, $\mathbb{E}\left[\dot{X}_{t} \dot{X}_{s}\right]=\delta(t-s)$ and $\mathbb{E}\left[X_{t}\right]=0$ ( $\mathbb{E}$ denotes the expectation under a reference probability). In general, the process $\left(X_{t}\right)$ can be a Brownian motion, a counting process or a mixing of both noises. Physically, the noise terms represent the output signal of the measurement and the probability of an event is traditionally described by $\left\|\psi_{t}\right\|^{2}$. Usually, asking $\left\|\psi_{t}\right\|^{2}$ to represent a probability density (giving the physical probability) implies $\mathbb{E}\left[\left\|\psi_{t}\right\|^{2}\right]=1$ and this last equality imposes the form of the operators $A$ and $B$. Finally, a new stochastic differential equation for normalized vector states $\left(\hat{\psi}_{t}\right)$ is derived. In the equation for the normalized states a new white noise appears, obtained by a suitable Girsanov transformation of the reference Brownian motion. A natural introduction of non-Markovian memory effects in the framework sketched above, can be obtained by replacing the white noise $\left(X_{t}\right)$ by a coloured Ornstein-Uhlenbeck noise.

To keep things simple here we consider a onedimensional Ornstein-Uhlenbeck process defined by $X_{t}=$ $\int_{0}^{t} \mathrm{e}^{-\gamma(t-s)} \mathrm{d} W_{s}$, where $\left(W_{t}\right)$ is a one dimensional Brownian motion, under a reference probability $\mathbb{Q}$. The Ornstein-Uhlenbeck process $\left(X_{t}\right)$ satisfies the stochastic differential equation

$$
\mathrm{d} X_{t}=-\gamma X_{t} \mathrm{~d} t+\mathrm{d} W_{t},
$$

where $\gamma>0$. The high non-Markovianity of the process is emphasized by the fact that the correlation function is not a pure delta function, but we have $\mathbb{E}_{\mathbb{Q}}\left[\dot{X}_{t} \dot{X}_{s}\right]=\delta(t-$ $s)-\frac{\gamma}{2}\left(\mathrm{e}^{-\gamma|t-s|}+\mathrm{e}^{-\gamma(t+s)}\right)$ and the Markovian regime is recovered in the limit $\gamma \downarrow 0$.

In the following, first we will show how this approach allows to reconstruct dynamical models with random Hamiltonians. Next, we will demonstrate how to construct a class of non-Markovian stochastic Schrödinger equations, that has an interpretation in terms of quantum measurement and unravelling.

Random Hamiltonians. Actually, the random Hamiltonian models arise naturally by translating the action of the Ornstein-Uhlenbeck process (2) in the definition of the linear stochastic Schrödinger equation (11). With the output driving process $\left(X_{t}\right)$ satisfying (2), Eq. (11) can be rewritten in the following form

$$
\mathrm{d} \psi_{t}=\left(A-\gamma X_{t} B\right) \psi_{t} \mathrm{~d} t+B \psi_{t} \mathrm{~d} W_{t},
$$

where the initial condition is a wave function $\psi_{0}$, such that $\left\|\psi_{0}\right\|^{2}=1$.

According to the laws of quantum measurement the probability of the output is described by the density $\left\|\psi_{t}\right\|^{2}$. This yields a measurement interpretation of Eq. (33). The quantity $\left\|\psi_{t}\right\|^{2}$ defines a probability density, if $\mathbb{E}_{\mathbb{Q}}\left[\left\|\psi_{t}\right\|^{2}\right]=1$, is satisfied for all times $t$. The consistency of these densities needs also the process $\left(\left\|\psi_{t}\right\|^{2}\right)$ to be a martingale (we refer to [19] for complete details). By Itô calculus rules, the stochastic differential of $\left\|\psi_{t}\right\|^{2}$ turns out to be

$$
\begin{array}{r}
\mathrm{d}\left\langle\psi_{t} \mid \psi_{t}\right\rangle=\left\langle\mathrm{d} \psi_{t} \mid \psi_{t}\right\rangle+\left\langle\psi_{t} \mid \mathrm{d} \psi_{t}\right\rangle+\left\langle\mathrm{d} \psi_{t} \mid \mathrm{d} \psi_{t}\right\rangle \\
=\left\langle\psi_{t} \mid\left[A^{*}+A-\gamma X_{t}\left(B^{*}+B\right)+B^{*} B\right] \psi_{t}\right\rangle \mathrm{d} t \\
+\left\langle\psi_{t} \mid\left(B^{*}+B\right) \psi_{t}\right\rangle \mathrm{d} W_{t} .
\end{array}
$$

In order to ensure that $\mathbb{E}_{\mathbb{Q}}\left[\left\|\psi_{t}\right\|^{2}\right]=1$, for all times $t$, we must impose that the term in $\mathrm{d} t$ must be equal to zero (and this guarantees also the martingale property). Indeed, in this way, we get $\mathbb{E}_{\mathbb{Q}}\left[\left\|\psi_{t}\right\|^{2}\right]=\mathbb{E}_{\mathbb{Q}}\left[\left\|\psi_{0}\right\|^{2}\right]=1$, since we have $\mathbb{E}_{\mathbb{Q}}\left[\mathrm{d} W_{t}\right]=0$. It follows that we must have

$$
A^{*}+A-\gamma X_{t}\left(B^{*}+B\right)+B^{*} B=0, \quad \forall t .
$$

This imposes that there are two self-adjoint operators $K$ and $H$ such that $B=-\mathrm{i} K$ and $A=-\mathrm{i} H-\frac{1}{2} K^{2}$. Then, Eq. (3) becomes

$$
\mathrm{d} \psi_{t}=\left[-\mathrm{i}\left(H-\gamma X_{t} K\right)-\frac{1}{2} K^{2}\right] \psi_{t} \mathrm{~d} t-\mathrm{i} K \psi_{t} \mathrm{~d} W_{t} .
$$

Let $\mathrm{T}_{\leftarrow}$ denotes the time ordering exponential, the formal solution of Eq. (6) is given by

$$
\psi_{t}=\mathrm{T}_{\leftarrow}\left\{-\mathrm{i} \int_{0}^{t}\left(H-\gamma X_{s} K\right) \mathrm{d} s-\mathrm{i} \int_{0}^{t} K \mathrm{~d} W_{s}\right\} \psi_{0} .
$$

In other terms, the evolution of the quantum system is completely determined by the time-dependent, random Hamiltonian $\hat{H}_{t}=H+\left(\dot{W}_{t}-\gamma X_{t}\right) K$ (a formal expression, due to the presence of $\dot{W}_{t}$ ). Note that Eq. (4) gives $\mathrm{d}\left\|\psi_{t}\right\|^{2}=0$, in agreement with a purely Hamiltonian evolution. This shows, that the usual measurement interpretation of (1) coloured with an Ornstein-Uhlenbeck process gives raise to a random Hamiltonian evolution. The property $\left\|\psi_{t}\right\|^{2}=1$ implies that we have not extracted information according to our measurement interpretation. We recover the framework of the evolution of an isolated closed system incorporating a random environment characterized in terms of Ornstein-Uhlenbeck noise.

In order to investigate the property of Eq. (6) in terms of unravelling, it is interesting to consider the evolution of the corresponding density matrices. To this end, we consider the pure state process $\left(\rho_{t}\right)$ defined by $\rho_{t}=\left|\psi_{t}\right\rangle\left\langle\psi_{t}\right|$. 
We define also the mean state $\eta_{t}=\mathbb{E}_{\mathbb{Q}}\left[\rho_{t}\right]$. Using Itô rules, the process $\left(\rho_{t}\right)$ satisfies the stochastic differential equation (SDE)

$$
\begin{aligned}
\mathrm{d} \rho_{t}=-\mathrm{i}\left[H-\gamma X_{t} K, \rho_{t}\right] \mathrm{d} t-\mathrm{i}[ & \left.K, \rho_{t}\right] \mathrm{d} W_{t} \\
& -\frac{1}{2}\left[K,\left[K, \rho_{t}\right]\right] \mathrm{d} t,
\end{aligned}
$$

which is, of course, equivalent to (6). Let us stress that the presence of the Ornstein-Uhlenbeck process implies that the solution $\left(\rho_{t}\right)$ of Eq. (77) is not a Markov process. For the mean state $\left(\eta_{t}\right)$ we have then

$$
\frac{\mathrm{d}}{\mathrm{d} t} \eta_{t}=-\mathrm{i}\left[H, \eta_{t}\right]-\frac{1}{2}\left[K,\left[K, \eta_{t}\right]\right]+\mathrm{i} \gamma\left[K, \mathbb{E}_{\mathbb{Q}}\left[X_{t} \rho_{t}\right]\right] .
$$

The above equation can be naturally considered as a Master equation, but its particularity is that it is not a closed equation for the mean state $\eta_{t}$. Actually, we have derived a model with memory for the mean state. Indeed, the term $\mathrm{i} \gamma\left[K, \mathbb{E}_{\mathbb{Q}}\left[X_{t} \rho_{t}\right]\right]$ introduces non-Markovian memory effects in the dynamics. Moreover, Eq. (6) is an unravelling of the Master equation (8).

Random coefficient The random Hamiltonian case we discussed above arose only because the coefficients $A$ and $B$ in the Eq. (11) were assumed to be not random. In the following we will address the situation, in which $A$ and $B$ are assumed to be functions of the Ornstein-Uhlenbeck process $\left(X_{t}\right)$, thus becoming random operators themselves. In other words, we consider now a SDE of the more general form

$$
\mathrm{d} \psi_{t}=A\left(X_{t}\right) \psi_{t} \mathrm{~d} t+B\left(X_{t}\right) \psi_{t} \mathrm{~d} X_{t} .
$$

Inserting the definition (2) of the Ornstein-Uhlenbeck process into Eq. (9) yields

$$
\mathrm{d} \psi_{t}=\left(A\left(X_{t}\right)-\gamma X_{t} B\left(X_{t}\right)\right) \psi_{t} \mathrm{~d} t+B\left(X_{t}\right) \psi_{t} \mathrm{~d} W_{t} .
$$

Again, asking the norm $\left\|\psi_{t}\right\|^{2}$ to be a probability density the condition (5) is translated into the following one

$$
\begin{aligned}
A^{*}\left(X_{t}\right)+A\left(X_{t}\right)-\gamma X_{t}\left(B^{*}\right. & \left.\left(X_{t}\right)+B\left(X_{t}\right)\right) \\
& +B^{*}\left(X_{t}\right) B\left(X_{t}\right)=0 .
\end{aligned}
$$

In contrast to the previous case with deterministic coefficients $A$ and $B$, we can now establish a relationship between the operators $A\left(X_{t}\right)$ and $B\left(X_{t}\right)$. More precisely, the condition (11) implies that there exists a self-adjoint operator $H\left(X_{t}\right)$ such that

$$
A\left(X_{t}\right)-\gamma X_{t} B\left(X_{t}\right)=-\mathrm{i} H\left(X_{t}\right)-\frac{1}{2} B^{*}\left(X_{t}\right) B\left(X_{t}\right) .
$$

Then, Eq. (10) becomes

$$
\begin{aligned}
\mathrm{d} \psi_{t}=\left(-\mathrm{i} H\left(X_{t}\right)-\frac{1}{2} B^{*}\left(X_{t}\right)\right. & \left.B\left(X_{t}\right)\right) \psi_{t} \mathrm{~d} t \\
& +B\left(X_{t}\right) \psi_{t} \mathrm{~d} W_{t} .
\end{aligned}
$$

In the general case $\mathrm{d}\left\|\psi_{t}\right\| \neq 0$ and the above evolution cannot be interpreted in terms of a random Hamiltonian. This is the signature that the above evolution describes the extraction of information from the system in terms of indirect quantum measurement. Indeed, the measurement interpretation relies upon the property that the norm $\|\psi\|^{2}$ defines a probability density. According to this property the relevant physical probability is defined by

$$
\mathbb{P}^{T}(\mathrm{~d} \omega)=\left\|\psi_{T}(\omega)\right\|^{2} \mathbb{Q}(\mathrm{d} \omega),
$$

where $\mathbb{Q}$ is the underlying reference probability as introduced in the definition of the Ornstein-Uhlenbeck process. In other words, the probability $\mathbb{P}^{T}$ is the physical probability law of the events which could occur in the time interval $[0, T]$. When the time $T$ varies, the probabilities $\mathbb{P}^{T}$ are "consistent", because $\left\|\psi_{T}\right\|^{2}$ is a "martingale" [19]. Consistency means that if we take $0<S<T$ and an event determined by conditions only in the time interval $(0, S)$, then $\mathbb{P}^{T}(F)=\mathbb{P}^{S}(F)$. Another important property of these probabilities is that they can be expressed in terms of quantum expectations on the premeasurement state of positive operator valued measures, as stated by the axioms of quantum mechanics [19].

In order to describe the stochastic dynamics of the system undergoing continuous indirect measurement we derive the corresponding stochastic Schrödinger equation for the normalized state vector $\widehat{\psi}_{t}=\psi_{t} /\left\|\psi_{t}\right\|$. Usually, such a process is called a quantum trajectory and satisfies a non-linear stochastic differential equation. To derive this equation we need to describe the driving random process in terms of the new physical probability $\mathbb{P}^{T}$. The famous Girsanov theorem gives that, under $\mathbb{P}^{T}$, the process

$$
\widehat{W}_{t}=W_{t}-\int_{0}^{t} m(s) d s
$$

with

$$
m(t)=\left\langle\widehat{\psi}_{t} \mid\left(B^{*}\left(X_{t}\right)+B\left(X_{t}\right)\right) \widehat{\psi}_{t}\right\rangle,
$$

is a new Brownian motion. This allows to express the output of the measurement $X_{t}$ (which was an OrnsteinUhlenbeck coloured noise under the reference probability $\mathbb{Q})$ in the form

$$
X_{t}=\int_{0}^{t} \mathrm{e}^{-\gamma(t-s)} m(s) \mathrm{d} s+\int_{0}^{t} \mathrm{e}^{-\gamma(t-s)} \mathrm{d} \widehat{W}_{s} .
$$

Moreover, by using Itô calculus we can write the nonlinear stochastic differential equation for the normalized state vector $\widehat{\psi}$ as

$$
\begin{aligned}
\mathrm{d} \widehat{\psi}_{t}=[ & \left.B\left(X_{t}\right)-\frac{m(t)}{2}\right] \widehat{\psi}_{t} \mathrm{~d} \widehat{W}_{t}-\left[\mathrm{i} H\left(X_{t}\right)+\frac{m(t)^{2}}{8}\right. \\
& \left.-\frac{m(t)}{2} B\left(X_{t}\right)+\frac{1}{2} B^{*}\left(X_{t}\right) B\left(X_{t}\right)\right] \widehat{\psi}_{t} \mathrm{~d} t
\end{aligned}
$$


Let us stress that the presence of the coloured noise $X_{t}$ in the above equation makes the process $\widehat{\psi}$ non-Markovian. Thus, Eq. (13) is an example of a non-Markovian stochastic Schrödinger equation that admits a clear interpretation in terms of indirect quantum measurement. Eq. (13) has the usual structure of a stochastic Schrödinger equation. Its particularity is the fact that the operators $H$ and $B$ are now random functions of the Ornstein-Uhlenbeck process $X_{t}$.

We shall investigate the evolution of the corresponding density matrix. To this end we define

$$
\widetilde{\rho}_{t}=\left|\widehat{\psi}_{t}\right\rangle\left\langle\widehat{\psi}_{t}\right|
$$

The non-linear stochastic master equation for the density matrix $\widetilde{\rho}_{t}$ takes the form

$$
\begin{array}{r}
\mathrm{d} \widetilde{\rho}_{t}=\mathcal{L}\left(X_{t}\right)\left[\widetilde{\rho}_{t}\right] \mathrm{d} t+\left[B\left(X_{t}\right) \widetilde{\rho}_{t}+\widetilde{\rho}_{t} B^{*}\left(X_{t}\right)\right. \\
\left.-\operatorname{Tr}\left\{\left[B\left(X_{t}\right)+B^{*}\left(X_{t}\right)\right] \widetilde{\rho}_{t}\right\} \widetilde{\rho}_{t}\right] \mathrm{d} \widehat{W}_{t},
\end{array}
$$

where

$$
\begin{aligned}
\mathcal{L}\left(X_{t}\right)[\rho]= & -\mathrm{i}\left[H\left(X_{t}\right), \rho\right] \\
& -\frac{1}{2}\left\{B^{*}\left(X_{t}\right) B\left(X_{t}\right), \rho\right\}+B\left(X_{t}\right) \rho B^{*}\left(X_{t}\right),
\end{aligned}
$$

defines a random Lindblad generator. Note, that again the originality relies on the fact that the operators $H$ and $B$ are random (see [12, 18] for similar considerations).

The mean state $\eta_{t}=\mathbb{E}_{\mathbb{P}^{T}}\left[\widetilde{\rho}_{t}\right]=\mathbb{E}_{\mathbb{Q}}\left[\left|\psi_{t}\right\rangle\left\langle\psi_{t}\right|\right]$ satisfies the following non-closed equation

$$
\frac{\mathrm{d}}{\mathrm{d} t} \eta_{t}=\mathbb{E}_{\mathbb{P}^{T}}\left[\mathcal{L}\left(X_{t}\right)\left[\widetilde{\rho}_{t}\right]\right]
$$

This equation describes a non-Markovian deterministic Master equation. Of course, by construction the process $\widehat{\psi}_{t}$ yields a non-Markovian unravelling of the Master equation (15).

A special case is featured by considering $B$ to be constant. In this case, we recover a model for the open system with a random Hamiltonian and a usual dissipative part in Lindblad form with a non random operator $B$.

In conclusion, a special class of non-Markovian stochastic Schrödinger equations and stochastic master equations has been derived. The framework of the traditional Markovian white noise theory has been extended by introducing the use of coloured Ornstein Uhlenbeck noise. This natural extension allows to describe random dynamics for open quantum system with memory effects. Two important categories of non-Markovian models has been derived. On the one hand, in a natural way, we have recovered the special case of random Hamiltonians. On the other hand, a generalization of Lindblad type equations has been obtained. The memory effects, influenced by the Ornstein Uhlenbeck noise, are encoded in the definition of the random operators which define either the Hamiltonian or the Lindblad operator. Our approach, based on the description of stochastic Schrödinger equations in terms of pure states, give rises to non-Markovian unravellings of these models.

\section{Acknowledgments}

This work is based upon research supported by the South African Research Chairs Initiative of the Department of Science and Technology and National Research Foundation.
[1] H-J Carmichael, Statistical Methods in Quantum Optics (Vol 1, Springer, Berlin 1999), (Vol 2, Springer, Berlin, 2008).

[2] C.-W. Gardiner and P. Zoller, Quantum Noise: A Handbook of Markovian and Non-Markovian Quantum Stochastic Methods with Applications to Quantum Optics, Springer Series in Synergetics (Springer, 2004).

[3] H.-P. Breuer and F. Petruccione, The Theory of Open Quantum Systems (Oxford University Press, Oxford, 2002).

[4] H.-P. Breuer and J. Piilo, EPL 85, 50004 (2009).

[5] H.-P. Breuer, Phys. Rev. A 75, 022103 (2007).

[6] M. Moodley and F. Petruccione, Phys. Rev. A 79, 042103 (2009).

[7] X. L. Huang, H. Y. Sun, and X. X. Yi, Phys. Rev. E 78, 041107 (2008).

[8] C. Pellegrini and F. Petruccione, Non Markovian Quantum Repeated Interactions and Measurements, J. Phys. A: Math. Theor., in press (October 2009).

[9] L. Diósi, N. Gisin and W. T. Strunz, Phys. Rev. Lett. 82, 1801 (1999).
[10] J. Gambetta and H. M. Wiseman, Phys. Rev. A 66, 012108 (2002).

[11] L. Diósi and W. T. Strunz, Phys. Lett. A 235, 569 (1997).

[12] D. Salgado and J. L. Sánchez-Gómez, J. Opt. B: Quantum Semiclass. Opt. 4, 458 (2002).

[13] J. Piilo, S. Maniscalco, K. Härkönen, and K.-A. Suominen, Phys. Rev. Lett. 100, 180402 (2008).

[14] J. Łuczka, Chaos 15, 026107 (2005).

[15] R. Kubo, M. Toda, and N. Hashitsume, Statistical Physics II. Nonequilibrium Statistical Mechanics (Springer, Berlin 1991).

[16] K. Wodkiewicz, Phys. Lett. A 84, 56 (1981).

[17] Ting Yu, J. H. Eberly Entanglement Evolution in a NonMarkovian Environment Special Issue of Optics Communications in Memory of Krzystof Wodkiewicz, eprint arXiv:0906.5378

[18] A. Budini, Phys. Rev E 72, 056106 (2005).

[19] A. Barchielli, M. Gregoratti, Quantum Trajectories and Measurements in Continuous Time - The diffusive case, Lecture Notes in Physics 782 (Springer, Berlin, 2009). 